\title{
¿Colonialismo biomédico o autonomía de lo local?: sanadores tradicionales contra la tuberculosis*
}

\section{Biomedical colonialism or local autonomy?: local healers in the fight against tuberculosis}

Antonio Miguel Ortega Martos

Doutorando do Departamento de Anatomía Patológica e Historia de la Ciencia/Facultad de Medicina/Universidad de Granada.

Facultad de Medicina/Universidad de Granada 18071 - Granada - España antoniom.ortega@gmail.com

Recebido para publicação em setembro de 2009. Aprovado para publicação em junho de 2010.
ORTEGA MARTOS, Antonio Miguel. ¿Colonialismo biomédico o autonomía de lo local?: sanadores tradicionales contra la tuberculosis. História, Ciências, Saúde - Manguinhos, Rio de Janeiro, v.17, n.4, out.-dez. 2010, p.905-924.

\section{Resumen}

Analiza el papel de los agentes médicos autóctonos y sus conocimientos en las campañas antituberculosas contemporáneas en el África subsahariana. Sitúa la medicina contemporánea, llevada a cabo en África en la herencia cultural de la medicina colonial, para comprender el marco histórico en el que se desarrollaron, a partir de los años setenta del siglo XX, las estrategias de la Organización Mundial de la Salud de promoción y desarrollo de las medicinas 'tradicionales'. En los proyectos sanitarios analizados, se evalúan las prácticas médicas locales y se entrenan a los agentes autóctonos para integrarlos en actividades estrictamente biomédicas: identificación de síntomas, remisión a hospitales o supervisión de tratamientos farmacológicos.

Palabras clave: sanadores tradicionales; tuberculosis; Organización Mundial de la Salud; África.

\section{Abstract}

The article explores the role played by indigenous medical agents, and their knowledge, within contemporary tuberculosis campaigns in sub-Saharan Africa. To understand the historical framework within which the World Health Organization devised its strategies to promote and develop traditional medicine as of the 1970s, the article contextualizes contemporary medicine as a cultural legacy of colonial medicine. Under the public healthcare projects analyzed in the article, local medical practices were assessed and indigenous agents trained so they could take part in strictly biomedical activities, like symptom identification, referrals to hospitals, or supervision of drug treatments.

Keywords: traditional healers; tuberculosis; World Health Organization; Africa. 
$\mathrm{C}$ onseguir unos niveles altos de salubridad en los países que son denominados como en desarrollo, pobres o del tercer mundo ha sido una tarea que históricamente y por distintas razones no sólo ha preocupado a los países afectados sino que también ha despertado interés internacional y se ha convertido en un reto para la biomedicina contemporánea. En la actualidad, la misión de expandir los medios para conseguir una salud global ha corrido a cargo de las agencias sanitarias internacionales mediante la gestión de lo que se ha definido como salud internacional, entendida como el fluir de asesoramiento, profesionales de la salud y tecnología sanitaria, desde los países ricos a los pobres (Rubinstein, Lane, 1990). La biomedicina contemporánea, cada vez más tecnificada, se ha visto limitada ante la imposibilidad de desarrollar sus recursos técnicos a escala mundial, máxime en zonas pobres, rurales o de difícil acceso. Ante esta limitación, la biomedicina comenzó, a finales del siglo pasado, a incluir el concepto de tradición en sus campañas de salud internacionales, enmarcándose dentro de un fenómeno relativamente nuevo como es el retorno de la cultura a las actividades biomédicas (Comelles, 2004).

¿Qué se entiende por tradición en el contexto de la salud internacional? ¿En qué sentido lo 'socio-cultural' ha llegado a formar parte de la industria del desarrollo en términos de salud? ¿Cómo son utilizados en la actualidad los saberes médicos autóctonos/locales/ indígenas? Para profundizar en estas interrogantes, he analizado una serie de campañas biomédicas internacionales que cuentan con lo 'tradicional' como eje vertebrador de sus actividades. En concreto, campañas de control de la tuberculosis (TBC), realizadas en el África subsahariana (entre 1996 y 2006) y que plantean como objetivo de su proyecto la integración de 'sanadores tradicionales' en sus actividades.

He elegido analizar las campañas porque explícitamente proponían recuperar a los practicantes médicos locales y sus saberes como agentes activos en las actividades antituberculosas. Por tanto, el objetivo de este trabajo será el análisis de este proceso de acercamiento de la biomedicina a lo local. Como fuente de información, he utilizado una serie de artículos publicados en revistas biomédicas, entre los años 1996 y 2006, en los que se da a conocer a la comunidad científica cómo se han realizado y qué resultados se han obtenido con los programas dirigidos a entrenar sanadores tradicionales.

Como trataré de argumentar, estas campañas no son actividades aisladas dentro de ciertos países subsaharianos, sino que forman parte de una estrategia global auspiciada por la Organización Mundial de la Salud (OMS) y otras agencias internacionales de promoción y desarrollo de las medicinas 'tradicionales' iniciada en los años setenta del siglo pasado. Esta situación implica que tanto el modelo de medicina tradicional como las actividades que se van a desarrollar en estas campañas provienen de las estrategias o políticas de la OMS en materia tradicional.

La política de la OMS de desarrollo de las medicinas tradicionales se ha caracterizado por una utilización de lo 'tradicional' como un recurso técnico dentro de sus campañas. Ha generado una serie de categorías (medicina tradicional, sanador tradicional) en las que los conocimientos médicos autóctonos se han desligado del contexto socio-cultural de la zona a intervenir y han sido relegados a una posición subalterna con respecto a las actividades biomédicas. Situación que puede explicar el fracaso de estas estrategias, ya que la información 
disponible demuestra las dificultades y fracasos de estas campañas cuando se han intentado implantar.

Para comprender las razones de este modelo de actuación es necesario contextualizar la medicina contemporánea en la herencia cultural de la medicina colonial. Como propone Packard (2000), la incapacidad de integrar sistemas indígenas de sanación demuestra la imposibilidad de romper con la herencia colonial. Por este motivo, comenzaré con un esbozo de lo que supuso la medicina colonial en el África subsahariana, después pasaré a describir cómo estos planteamientos pudieron influir en las estrategias de la OMS. Una vez contextualizado el fenómeno de integración de las medicinas tradicionales, pasaré a analizar el caso concreto de las campañas antituberculosas del África subsahariana que han contado desde su diseño con sanadores tradicionales.

\section{La medicina colonial, punto de partida de la salud internacional}

Si queremos comprender la situación actual de la salud en el África subsahariana, así como conocer la relación de los sistemas locales de salud con las agencias internacionales, necesitamos mirar a la historia y, en el caso africano, remontarnos al periodo colonial. Para ello utilizaré las aportaciones que desde la teoría crítica postcolonial se han hecho a la compresión de los factores sociales, económicos, políticos y culturales que han determinado el devenir, por un lado, de la salud en este continente y, por otro, su relación con Occidente. Esta relación debe ser entendida como la que se estableció en el periodo colonial con las agencias coloniales europeas y, tras la independencia de las colonias, la relación que se fraguó con las agencias occidentales de salud. Mediante la utilización de la teoría postcolonial, pretendo generar un marco de análisis crítico que trascienda el postcolonialismo como periodo histórico y lo enmarque dentro de una forma de abordar los problemas actuales del continente africano en relación al contexto histórico de la expansión de las colonias europeas y sus efectos actuales (Anderson, 2002). Como propone Silva (1994) para el caso de la erradicación del paludismo en Sri Lanka, asumiré que las crisis actuales en el control de las enfermedades contagiosas requieren un análisis no sólo de los programas y políticas actuales sino que es de primera necesidad introducir una perspectiva histórica.

En el África subsahariana precolonial y según las aportaciones de Feierman (1985), los sanadores populares, en la segunda mitad del siglo XIX, gozaban de un rol central en la organización y producción social y estaban estrechamente vinculados a las estructuras de control sociopolítico. Las prácticas de sanación solían estar relacionadas con las prácticas económicas, políticas o religiosas. Según estudios sobre la salud en África (Prins, 1991), la salud local se mantenía gracias a una relación armoniosa entre ser humano y ambiente. Los factores ecológicos formaban parte de la organización social y cultural de la vida cotidiana y, por tanto, de la búsqueda del bienestar. El sistema de control de la enfermedad basado en la relaciones socioambientales también tuvo sus deficiencias. De hecho, existieron catástrofes que asolaron la población africana. Igualmente se conoce la existencia de disputas por la autoridad entre los sanadores, lo que provocó luchas de clases por el control del mantenimiento de la salud. ${ }^{1}$ Trascendiendo las virtudes o deficiencias de estos sistemas 
diversos de organización social de la sanación, lo que nos interesa es su incrustación en el tejido social que hacía del sanador un líder con autoridad política y económica.

La colonización generó cambios en toda la estructura social de las zonas conquistadas y en las formas de afrontar la enfermedad. Si en la época precolonial los sanadores tenían poder dentro de la sociedad, con la inclusión de la gestión colonial ellos pasan a un segundo plano y son apartados de la política local. Este 'desapoderamiento' de la sanación tradicional transformó las prácticas y los sistemas de pensamiento de los sistemas de salud autóctonos (Feierman, 1985). Según Iliffe (1998), la colonización fue "tan traumática como breve" (p.271) y supuso un aumento demográfico que instaló a África en una nueva dinámica. Los africanos tuvieron que adaptarse a esta nueva situación mediante la génesis de nuevas identidades sociales y políticas que influyeron en las formas locales de gestionar la salud. La medicina colonial estuvo implicada en todas las fases de la colonización y formó parte del aparato ideológico del imperio (Arnold, 1993). Fue una medicina sustentada en los aspectos técnicos de la sanación, centrada en los intereses económicos de los colonizadores y que sirvió como justificación de la empresa colonial.

La nueva reorganización del mapa político y económico africano trajo consigo una serie de cambios que influyeron en la salud. Así, en Sudáfrica, la exportación del modelo industrial europeo, a finales del siglo XIX, produjo cambios estructurales como el aumento de migraciones, fundamentalmente de las zonas rurales a las urbanas, cambios en las condiciones laborales o la instauración de trabajos que afectaban negativamente a la salud (Packard, 1992). Estos cambios se tradujeron en un aumento de las parasitosis, malnutrición y hacinamiento que provocaron una mayor incidencia de ciertas enfermedades como la silicosis y la neumonía que, a la vez, se agravaron por una escasa asistencia sanitaria. Por tanto, la colonización provocó una desestructuración ecológica (ambiental y humana) que aumentó la prevalencia de ciertas enfermedades, como el paludismo en Guinea Ecuatorial (Medina, 2003) o el tifus en Sudáfrica (Marks, Anderson, 1988).

El sistema sanitario colonial se centró en la fuerzas de producción y en la salud de los colonizadores y generó un sistema de sanación desigual que dejó fuera de cobertura a gran parte de la población rural, a las mujeres, a los niños y a la población empobrecida, desarrollando una asistencia selectiva que Arnold (1993) ha denominado "en enclaves" 2 que afectó de manera particular a las mujeres. Es indicativo que en Nigeria en 1930 dos tercios de la gente asistida en los hospitales fueran hombres (Iliffe, 1998, p.304-309).

En la segunda mitad del siglo XX, con la progresiva descolonización, hubo cambios significativos que provocaron mejoras en la asistencia sanitaria. Pero predominó un modelo de asistencia que relegaba los sistemas indígenas y que vinculaba sanidad con costes de producción, centrándose fundamentalmente en un desarrollo de tecnologías médicas que pudieran mejorar la situación sanitaria local. Las agencias internacionales adquirieron estas pautas de actuación, lo que supuso una reproducción de los patrones coloniales sustentada en la reafirmación del modelo médico occidental. Por tanto, tecnología, política y economía fueron los cimientos de las propuestas foráneas en la configuración de los sistemas médicos locales. La independencia de los estados coloniales pudo suponer un cambio en los servicios sanitarios pero la realidad fue distinta. La impronta del modelo de gestión occidental, junto con los ideales renovadores nacionalistas, provocaron que se 
adquirieran los modelos de desarrollo occidentales en la economía y en la sanidad en países tan diversos como Mozambique, Zaire o Somalia (Illife, 1998, p.328-336).

Las campañas sanitarias que se llevaron a cabo en los años sesenta y setenta, como los programas para combatir el paludismo, sarampión y tuberculosis o programas orientados a las vacunaciones o planificación familiar, se caracterizaron por ser medidas ancladas en una organización piramidal o lo que se conoce como programas verticales (Van der Geest, Speckmann, Streefland, 1990). Es decir, programas desarrollados en su totalidad desde Occidente por las potencias y agencias internacionales y que se ejecutaban sin contar con representación local. La dependencia ahora no era tanto del gobierno local, sino de las agencias internacionales de desarrollo que se erigieron en las instituciones que deben velar por la salud de la, aún considerada, África enferma. El cambio más importante fue que los centros de control político de la salud viran desde los gobiernos coloniales locales hacia los nuevos centros de decisión (situados en países occidentales sedes de las agencias internacionales), pero se siguen reproduciendo patrones coloniales tales como falta de representación local, paternalismo (la población africana es vista como incapaz de solventar sus problemas), entronización y universalización de la biomedicina y definición de la salud como un medio para el desarrollo económico y social más que un fin en sí mismo (Medina, 2005, p.91). Como han señalado algunas voces críticas desde dentro de la propia organización, con la buenas intenciones no bastaba (Ruiz-Giménez, 2003) ya que las intervenciones pro-desarrollo seguían sustentadas en una estructura ideológico-gerencial que no permitió una adaptación a las realidades locales. Como expondré a continuación, las agencias de salud internacionales quisieron cambiar el tipo de asistencia para adaptarse a las nuevas realidades globales en la década de los setenta del siglo XX. Pero la fuerte herencia colonial así como los principios teóricos y prácticos biomédicos determinaron el tipo de asistencia que se iba a extender.

\section{El marco institucional de desarrollo de lo tradicional: las estrategias de la OMS sobre las medicinas tradicionales}

Veíamos con anterioridad como los principios de asistencia biomédica y los patrones coloniales caracterizaron el modus operandi de las primeras actividades sanitarias realizadas por agencias internacionales tras la descolonización. Ante el fracaso de estas campañas en los años setenta hubo un intento por parte de ciertas instituciones internacionales, destacando la OMS, de cambiar de asistencia ofrecida a los países desfavorecidos.

Esta propuesta de cambio se debió en gran medida al doctor Haldan Mahler, director general de la OMS desde 1973 hasta 1988. Mahler, que había trabajado en el programa nacional de control de la tuberculosis en la India, orientó las políticas de la OMS hacia el desarrollo de servicios sanitarios globales que actuaran sobre la base de los problemas locales de salud y que contasen con la población autóctona. Esta idea se materializó en forma de estrategia en 1978 con la Declaración de Alma Ata (WHO, Unicef, 1978). Allí los expertos asentaron las bases de lo que iba a ser la "atención primaria de salud", entendida como un sistema de salud universal, basado en la comunidad y donde el hospital no fuera el centro. Conceptos como integración horizontal de los cuidados sanitarios, potenciación 
de las medidas preventivas o salud global comenzaron a aparecer en las agendas de los gestores de la salud. En este contexto se planteó rescatar a los agentes locales como activos en su propia sanación y se pensó en las medicinas tradicionales y sus representantes como colaboradores en la lucha contra enfermedades infecciosas. Como veremos con posterioridad en el análisis de las campañas antituberculosas, las estrategias, políticas o recomendaciones que derivarían de estas medidas estaban destinadas a ser los principios rectores de la asistencia sanitaria auspiciada por la OMS en materia tradicional hasta la actualidad. Mediante la definición de qué y cómo se deben promocionar y desarrollar las medicinas tradicionales, la OMS comenzó a marcar la forma de abordar los sistemas médicos tradicionales por parte de los agentes biomédicos.

La OMS respondió a las necesidades generadas tras Alma Ata con un plan de acción orientado a la promoción y desarrollo de las medicinas tradicional: The promotion and development of traditional medicine (WHO, 1978). Este informe establecía pautas prácticas para fomentar la colaboración entre los distintos sistemas de salud, la formación del personal encargado de prestar atención sanitaria, la promoción de la medicina tradicional e investigación sobre sus prácticas (evaluación de la nocividad) e integración en los servicios nacionales de salud. Según el informe de 1978, la medicina tradicional debía ser promovida por su bajo costo, su aceptación por parte de la comunidad, por dar solución a problemas relacionados con el contexto socio-cultural, así como por el enfoque holístico en la visión del ser humano.

Sin embargo, lo que parecía ser un reconocimiento y potenciación de las prácticas médicas tradicionales como sistema de sanación autónomo, capaz de solventar los problemas de salud locales, se planteó finalmente como una adaptación de los agentes locales a las premisas de la medicina occidental. Cuando profundizamos en el análisis del informe de 1978, la idea programática de diálogo entre saberes médicos comienza a tomar forma en la de 'integración' de las medicinas tradicionales dentro del sistema biomédico como respuesta a la escasez de recursos biomédicos.

Merece la pena detenerse en dos conceptos que aparecen en la estrategia de la OMS y que determinaron la forma del acercamiento o reconocimiento de esta institución a los programas dependientes de los saberes médicos autóctonos o locales en los denominados países en desarrollo: medicina(s) tradicional(es) e integración. Como aparece en el informe de 1978, la OMS definió la medicina tradicional como "la suma de todos los conocimientos teóricos y prácticos, explicables o no, utilizados para el diagnóstico, prevención y supresión de trastornos físicos, mentales o sociales, basados exclusivamente en la experiencia y la observación, y transmitidos verbalmente o por escrito de una generación a otra" (WHO, 1978, p.8).

Tal y como se ha defendido desde ciertas posturas críticas ${ }^{3}$, en el caso de la estrategia de 1978, la OMS utilizó una idea de medicina tradicional reduccionista y homogenizante. No se distinguió entre las distintas formas de sanación, se pasó por alto los contextos locales y se definió y categorizó a los saberes médicos autóctonos según principios propios de la biomedicina como son el diagnóstico o la prevención. Las prácticas tradicionales, según el informe de 1978, estaban ancladas en la experiencia y la observación, por tanto compartían la característica de ser acientíficas. Al resaltar la posibilidad de que los conocimientos no 
fueran 'explicables', nos muestran los presupuestos occidentales que inspiraban el informe al destacar la necesidad de dar coherencia explicativa a las practicas humanas desde ciertos a priori explicativos o desde una conceptualización de lo que constituye una explicación.

Una vez que se definió en el discurso de las agencias internacionales el objeto de estudio y por tanto la realidad intangible, heterogénea y poliédrica de los conocimientos y prácticas locales en salud se etiquetó con una categoría hermética y acotada como es el concepto medicina tradicional. Pero cabe preguntarse ¿Qué alcance tuvo esta definición? ¿Se fomentó un empoderamiento de lo local y un respeto y reconocimiento de los saberes autóctonos? ¿Evaluó lo OMS la eficacia simbólica de las prácticas indígenas e intentó comprender el marco cultural en el que cobran sentido las diferentes medicinas tradicionales y sus practicantes?

Para comprender el proceso de desarrollo de las medicinas tradicionales planificado por la OMS debemos analizar el concepto de integración. Para la OMS, los objetivos planteados en su estrategia debían ser conseguidos mediante la integración del sistema biomédico y del tradicional. Como aparece en el informe: "La integración efectiva, como lo que se ha logrado en China, entraña una síntesis de las ventajas de la medicina tradicional y la llamada medicina 'occidental' mediante la aplicación de conocimientos y métodos científicos modernos" (WHO, 1978, p.17).

Basándose en el modelo chino la OMS intentó solventar los fallos de las campañas sanitarias internacionales con un plan de acción sustentado en la evaluación científica de lo tradicional. El sistema chino de los denominados médicos descalzos se basó en la occidentalización o modernización de la medicina tradicional china. Los médicos descalzos fueron entrenados, en las décadas de los sesenta y setenta del siglo XX, en los principios de la asistencia biomédica (medidas higienistas, prevención, diagnóstico precoz, etc.) y consiguieron mejorar las condiciones sanitarias de distintas zonas de China, fundamentalmente rurales, donde no llegaba el sistema sanitario oficial. El modelo chino fue, por tanto, la fuente de inspiración de la OMS y la base de sus propuestas de integración que habrían de extenderse a otros continentes. Sin embargo, la efectividad, autoridad y reconocimiento de este modelo de asistencia sanitaria cobró sentido en un contexto particular, lo que hacía difícil su traslación mimética a territorios como el africano. Como ha mostrado Francesca Bray (1993), los cambios socio-políticos influyeron directamente en la legitimidad y reconocimiento de la medicina china. Un régimen autoritario como el maoísta propició el cumplimiento con contundencia de sus medidas político-sociales. En contraposición, en zonas como el África subsahariana existe una gran variabilidad local (política, social y cultural). Además, a diferencia del modelo de asimilación chino, el territorio africano no cuenta con una medicina tradicional 'subsahariana', dotada de un corpus de conocimientos institucionalizado y con cierta homogeneidad en las prácticas y creencias. ${ }^{4}$

Podemos concluir que el plan de acción de la OMS pasó por alto el modelo sociocultural en el que cobraban sentido los conocimientos locales. Se centró en la evaluación científica de las prácticas tradicionales para un posterior 'readiestramiento profesional' de los practicantes médicos locales o sanadores tradicionales, como son etiquetados por la OMS, aunque sus funciones sean las de un recurso asistencial biomédico. Lo sociocultural 
quedó oscurecido por una idea de medicina tradicional que no fue utilizada como un fin en sí misma sino como una adaptación de lo local o autóctono a la ontología y la epistemología biomédicas. Como defiende Packard (2000), el propio término de integración decía muy poco de los practicantes de la medicina tradicional y mucho de las capacidades que los occidentales querían desarrollar en ellos para ser considerados 'aptos' o 'capacitados' para desempeñar funciones dentro de las campañas internacionales de desarrollo.

Las reacciones frente a la propuesta de la OMS de 1978 fueron muy variadas y dependieron de los gobiernos donde se pretendía aplicar, de los agentes biomédicos, de los sanadores locales o de la propia población autóctona. En el caso africano, no todos los gobiernos estaban dispuestos a colaborar en la promoción de la medicina tradicional (Freeman, Motsei, 1992). Y hubo diferencias significativas nacionales en el proceso de 'profesionalización' de los sanadores (Last, 1990).

Como ocurrió en América Latina (Menéndez, 1996), en África hubo reacciones ambiguas de los sanadores a la vinculación con los médicos occidentales. Como defiende McMillen (2004), los sanadores cambian sus roles al entrar en contacto con la tecnología biomédica. Incluso sus prácticas se pueden ver modificadas para demostrar la validez y eficacia de sus tratamientos tradicionales. Por otro lado, hay casos descritos en los que los agentes locales rechazaron la biomedicina o bien porque suponía una expropiación de su parcela de actuación o por la dificultad de coexistir y ajustarse al paradigma biomédico (Offiong, 1999).

Además, el grado de adaptabilidad de los agentes biomédicos a trabajar con su nuevos 'aliados sanitarios' fue variado. Como señala Green (1988), ciertos equipos médicos rechazaron la posibilidad de colaborar con sanadores locales. Tal fue el caso de colaboración en el control de enfermedades diarreicas en Suazilandia (Green, Makhubu, 1984), donde las prácticas tradicionales (administración de enemas, inducción del vómito o tratamientos herbarios) fueron calificadas por los responsables del proyecto como nocivas y, por tanto, debían ser cambiadas.

Estas reacciones de rechazo junto a la distancia cultural existente entre profesionales biomédicos y sanadores, así como la carencia de personal biomédico que supervisara las tareas, obstaculizaron la consecución de los objetivos propuestos por la OMS (King, 2000). Pero la idea de promocionar las medicinas tradicionales continuó formando parte de las principales líneas estratégicas de la OMS, especialmente en el África subsahariana, a pesar de que los resultados no eran positivos y satisfactorios. En el estudio realizado en 1991 (WHO, 1991), un grupo de expertos revisaron 17 proyectos que habían incorporado sanadores tradicionales (incluyendo parteras empíricas) como trabajadores de salud comunitarios (community health workers). Tras revisar los 17 proyectos, el trabajo concluía que, a pesar de algunos aspectos positivos, como la disponibilidad y utilidad de los sanadores para trabajar en la atención comunitaria, existían muchas limitaciones. El estudio resaltó como obstáculos fundamentales el déficit de políticas locales para la cooperación, la nocividad de ciertas prácticas tradicionales y la dificultad de cambiarlas, los conflictos entre sanadores y agentes biomédicos, las deficiencias en la definición de los roles de los sanadores y la falta de evaluación de sus conocimientos. En años posteriores, otras agencias internacionales como el Programa Conjunto de las Naciones Unidas Sobre el VIH/Sida (Onusida) también evaluó el papel de sanadores tradicionales en proyectos centrados en el control del Sida. ${ }^{5}$ 
Tras el acúmulo de pruebas sobre el modelo inspirado en el documento de 1978, en 2002 la OMS emitió una estrategia global sobre medicina tradicional: WHO traditional medicine strategy 2002-2005 (WHO, 2002) que trataba de subsanar los problemas surgidos. El informe proponía una estrategia a seguir entre 2002 y 2005 en la que "se maximice el potencial de la medicina tradicional y complementaria o alternativa como fuente de cuidado de la salud" (p.3). Fue elaborada desde la sede central y el personal de las oficinas regionales de la OMS del Departamento de Medicamentos Esenciales y Política Farmacéutica, en colaboración con personal de otros programas. También participaron algunas ONG, la ONU, organizaciones internacionales y centros de medicina tradicional que colaboraban con la OMS.

Los expertos sugirieron cuatro puntos clave en el desarrollo de la medicina tradicional. Primero, elaborar políticas sanitarias nacionales e internacionales concretas. Segundo, aumentar la seguridad, eficacia y calidad de las medicinas tradicionales mediante el análisis científico de sus productos y prácticas. Tercero, mejorar el acceso, sobre todo en los países en vías de desarrollo, a los sistemas de sanación locales. Y, finalmente, hacer un "uso racional" de ellos (WHO, 2002, p.4). Sin embargo, la estrategia de 2002 muestra pocos cambios de fondo respecto a la de 1978. La OMS continuó con la idea de generar una medicina tradicional a imagen y semejanza de la biomedicina, sustentada en la profesionalización de los agentes al estilo biomédico 'oficial' y la comprobación científica de la eficacia bioló-gica de las prácticas autóctonas.

Como trataré de argumentar a continuación, con el análisis de las campañas antituberculosas contemporáneas que contaron con sanadores tradicionales, la OMS volvió a plantear una estrategia que reprodujo, en gran medida, patrones procedentes del programa de 1978. La evaluación científico-médica de lo tradicional para la posterior integración de las medicinas tradicionales en el aparato biomédico continuó siendo el principio regidor de la estrategia de la OMS y proyectos dependientes.

\section{La colaboración con sanadores(as) tradicionales en las campañas de control de la tuberculosis en el África subsahariana}

Para mi análisis he utilizado una serie de proyectos realizados en el África subsahariana en los que se han evaluado las practicas tradicionales relacionadas con la TBC y cuyo objetivo ha sido valorar la idoneidad de contar con sanadores tradicionales como agentes útiles en el control de esta enfermedad. He estudiado los proyectos a partir de los artículos publicados en revistas biomédicas. Tras restringir la búsqueda a artículos centrados en la colaboración con sanadores tradicionales en el control de la TBC, realizados en el África subsahariana, bajo responsabilidad del personal biomédico y publicados entre 1996 y 2006, he localizado y analizado 14 artículos: Banerjee et al., 2000; Brouwer et al., 1998; Colvin et al., 2003; Edginton, Sekatane, Goldstein, 2002; Godfrey-Faussett, Ayles, 2003; Harper et al., 2004; Peltzer, Mngqundaniso, Petros, 2006a; Peltzer, Mngqundaniso, Petros, 2006b; Pronyk et al., 2001; Salaniponi et al., 2000; Sanou et al., 2004; Steen, Mazonde, 1998; Steen, Mazonde, 1999; Wilkinson, Gcabashe, Lurie, 1999. De estos artículos, seis corresponden a proyectos realizados 
en la República Sudafricana, tres en Malawi y el resto en zonas como Gambia, Burkina Faso y Botswana.

En estos artículos se exponen las principales características de las campañas así como una evaluación científica de las actividades que se realizaban. Sin embargo, debido a la estructura restrictiva de las publicaciones científicas, no aparecen descritas en profundidad todas las actividades realizadas. A pesar de estas limitaciones, he comparado las directrices que marca la OMS en su política sobre las medicinas tradicionales con las actividades realizadas en las campañas contra la TBC en el África subsahariana y he caracterizado el tipo de integración de sanadores en los programas sanitarios internacionales. Finalmente, a través de los artículos publicados, he analizado la idea de que las instituciones que trabajan en el desarrollo sanitario manejan las medicinas tradicionales, sus practicantes y su integración en los sistemas biomédicos.

Estos proyectos se enmarcan dentro de una política de la OMS, iniciada en los años noventa, dirigida a disminuir la incidencia y prevalencia mundial de la TBC y reducir la mortalidad asociada al contagio por el bacilo (Kochi, 1991). La política de la OMS se ha basado en la búsqueda de casos nuevos y en la instauración de tratamiento antituberculoso, fundamentalmente mediante el programa Dots (Directly Observed Treatment Short-Course). A grandes rasgos, este programa se basa en la extensión global de servicios sanitarios contra la TBC, para así poder diagnosticar más casos e instaurar el tratamiento antituberculoso. Este tratamiento consiste en la administración de una combinación diaria de antibióticos durante unos seis-ocho meses. Ante la escasez de personal biomédico que pueda supervisar dichas tareas, la OMS, en consonancia con el programa sobre medicina tradicional, ha elaborado una estrategia de captación de sanadores autóctonos como 'aliados' en su lucha contra el bacilo tuberculoso. Por tanto, como trataré de argumentar en esta sección, en estos proyectos son escasas las medidas orientadas a la comprensión del modelo cultural en torno a la sanación en cada zona intervenida. Las intervenciones están dirigidas al entrenamiento de sanadores autóctonos en el desarrollo de habilidades, conocimientos y prácticas para identificar síntomas, supervisar tratamientos y remitir a los hospitales a personas con TBC. Los proyectos analizados no contemplan cuestiones como la identificación de los factores socioculturales que influyen en la distribución de la tuberculosis o la importancia de los saberes médicos locales dentro de la comunidad o el rol que tiene el sanador entre la población, por nombrar algunos de los aspectos que pueden influir en la comprensión de otros modelos explicativos de la enfermedad distinto al biomédico, o si lo hacen, como veremos en lo que se propone en algunos trabajos, se hace de forma tamizada.

Los artículos analizados pueden agruparse en dos grandes conjuntos: en primer lugar, trabajos que se han centrado en la evaluación de la colaboración con sanadores tradicionales en campañas antituberculosas (Banerjee, 2000; Brouwer, 1998; Colvin et al., 2003; Edginton, Sekatane, Goldstein, 2002; Godfrey-Faussett, Ayles, 2003; Harper et al., 2004; Peltzer, Mngqundaniso, Petros, 2006a, 2006b; Wilkinson, Gcabashe, Lurie, 1999) y en segundo lugar, estudios que han analizado los factores, fundamentalmente las conductas y creencias de la población local, que influyen en el diagnóstico y tratamiento de la tuberculosis (Pronyk et al., 2001; Salaniponi et al., 2000; Sanou et al., 2004; Steen, Mazonde, 1998, 1999). Por tanto, la intencionalidad de los trabajos se nos muestra clara. Aunque se plantean 
como un intento de recuperar los conocimientos locales o autóctonos de sanación, los objetivos reales sobre el terreno son el análisis científico tanto del propio proceso de colaboración como de las creencias y conductas autóctonas para que mediante programas de entrenamiento se consiga 'corregir' comportamientos erróneos e inculcar ciertos conocimientos biomédicos para que los agentes locales puedan formar parte de las propias campañas.

Aunque los artículos son producto de una investigación sobre programas específicos, estas investigaciones también forman parte de proyectos generales. En las publicaciones analizadas no aparece necesariamente el proyecto general en el que se enmarca la investigación, pero al aparecer la agencia financiadora o la adscripción institucional de los autores podemos inferir qué organizaciones o qué programas sustentan estas investigaciones.

La financiación de los proyectos procede, fundamentalmente, de dos fuentes: ayudas de agencias internacionales, principalmente la OMS, y de los gobiernos nacionales a través de sus programas de control de la tuberculosis, como el Programa Nacional de Control de la Tuberculosis (NTCP, en inglés) de Malawi y Burkina Faso, el Programa de Sudáfrica (TBCP, en inglés), o del Departamento de Control de la Tuberculosis en Gambia. La OMS apoya institucionalmente estos proyectos mediante el programa Dots y aparecen también otras agencias internacionales como la International Union Against Tuberculosis and Lung Desease (IUATLD, en inglés). Merece la pena profundizar en el análisis de estas agencias y sus campañas para conocer el engranaje organizativo que hay tras estos proyectos y los principios rectores de las actividades realizadas. Como veremos a continuación, las agencias que intervienen en estos proyectos se encuentran íntimamente relacionadas $y$, aunque parten de los programas nacionales de control de la $\mathrm{TBC}$, finalmente todos entran dentro del programa de la OMS de control de la TBC y más en concreto del ya nombrado Dots.

La IUATLD, también llamada The Union, es una agencia internacional creada en 1920 en París con el objetivo de luchar contra la TBC a nivel mundial. La colaboración con la OMS comenzó en 1946 y ha perdurado hasta la actualidad. Cabe destacar que la IUATLD comenzó en 1961 a elaborar proyectos de asistencia técnica a países en desarrollo mediante la trasferencia de tecnologías médicas para el control de la TBC, en 1978 participó en Alma Ata y desde 1978 ha estado estrechamente vinculada a la estrategia Dots y ha influido directamente, dando apoyo institucional, en los programas nacionales antituberculosos tanto de países africanos como asiáticos. En 1993, la OMS incluyó en las recomendaciones de la estrategia "Stop tuberculosis" el modelo de actuación de The Union.

Otras instituciones distintas que también han colaborado o son responsables de algunos proyectos analizados, como los programas cuyos resultados publican Peltzer, Mngqundaniso y Petros (2006a, 2006b), provienen de otros ámbitos como las ciencias sociales o las industrias farmacéuticas. Los autores de este trabajo pertenecen al Human Sciences Research Council de Sudáfrica, centro especializado en el estudio de los determinantes sociales en salud y centrado en el análisis social del Sida. A la vez cuenta con la financiación de la empresa farmacéutica Bristol-Myers a través de su fundación para el control del Sida en África (Bristol-Myers Squibb Foundation). A pesar de que en este proyecto parece que lo social ocupa un lugar central en la planificación de la investigación, según la información extraída 
del artículo, el patrón de acción/investigación es semejante a los demás. Los autores analizaron los conocimientos, creencias y prácticas de los sanadores tradicionales en KwaZulu-Natal (Sudáfrica) y el cambio de los mismos tras un proceso de entrenamiento.

Se trata, por tanto, de un marco de cooperación internacional entre las agencias externas, fundamentalmente la OMS y los gobiernos nacionales, aunque con permisividad por parte de los gobiernos locales ante las directrices que marca la OMS en el control de la TBC. Con permisividad me refiero a que las pautas de actuación, la financiación, así como el personal encargado de los proyectos corren a cargo de las agencias internacionales. Esta organización ha facilitado que la política llevada a cabo en el reconocimiento de los sanadores como agentes de control de la TBC esté basada en las estrategias de promoción y desarrollo de la medicina tradicional de la OMS. Por tanto, las pautas de investigación sobre los sanadores, métodos de entrenamiento, creación de categorías a intervenir (sanador tradicional, medicina tradicional, etc.) o el modo de integración propuesto, responde, como veremos a continuación, a la idea oficial sobre las medicinas tradicionales que subyace en el discurso del desarrollo que encontramos en las políticas de intervención de la OMS desde finales de los setenta.

\section{El análisis científico de lo tradicional}

Para comprender la forma de colaboración entre los sanadores tradicionales y el personal biomédico en el control de la TBC así como las prácticas, relaciones y discursos producto de la interacción, utilizaré el esquema que describe Stacy Pigg (1995) en su análisis del proceso de entrenamiento de los denominados practicantes médicos tradicionales realizado en los años setenta y ochenta del siglo XX en Nepal. La autora plantea que las prácticas de entrenamiento de los agentes locales pasan por tres fases: la primera, consistiría en identificar a los actores locales que se ajusten a la categorías propuestas desde el discurso occidental del desarrollo (sanadores tradicionales, parteras empíricas, practicantes indígenas, etc.); la segunda, generar y extender un nuevo conocimiento médico que se ajuste al contexto local; y la tercera, alinear las prácticas y roles locales con las categorías definidas por el modelo médico.

Como ha señalado Escobar (1992), la planificación del desarrollo requiere la normalización y la estandarización de la realidad, lo que a su vez implica la extinción de la diferencia y diversidad. En el caso de las intervenciones de los proyectos analizados, mediante la categorización del agente sanador(a) tradicional, se intenta homogenizar la diversidad de las prácticas locales para la búsqueda de salud. Primero, se define qué es o más bien qué debe ser un sanador tradicional, siguiendo el modelo predefinido en los informes, estrategias o políticas de la OMS sobre las denominadas medicinas tradicionales. Una vez que se define el objeto de intervención, se pasa a identificar qué agentes locales se corresponden con la categoría definida como sanador tradicional. En los textos analizados se utiliza el término sanador tradicional sin particularizar las características locales, ya sean los programas centrados en Sudáfrica como los de Gambia, Malawi o Bostwana. Una vez definido lo que debe ser un sanador, los programas identifican a los agentes $y$, finalmente, evalúan sus prácticas. 
El proceso de búsqueda de los practicantes médicos tradicionales suele hacerse, en la mayoría de los casos, mediante la consulta de los registros que suelen tener las asociaciones locales de sanadores tradicionales. Tan sólo en el proyecto de Banerjee (2000) se consultó también a líderes locales o trabajadores sanitarios comunitarios. Banerjee et al. (2000), Colvin et al. (2003), Harper et al. (2004; en este caso se utilizó un registro del gobierno), Peltzer, Mngqundaniso, Petros (2006a, 2006b) y Wilkinson, Gcabashe, Lurie (1999) utilizaron registros oficiales de sanadores. Al utilizar registros oficiales o asociaciones para localizar a los sanadores, quedan fuera todas aquellas personas que se dedican a las prácticas de sanación autóctonas y que no han sido recogidas en estos registros o no pertenecen a asociaciones. En las prácticas medicas autóctonas, las actividades para mejorar la salud no siempre cuentan con una figura central exclusivamente dedicada a la profesión de sanador (Knipper, 2006). Esta idea de 'profesionalización' de las medicinas tradicionales atiende más al discurso sobre la integración de las agencias internacionales que a las realidades socioculturales locales. Last (1990) defiende que mediante la profesionalización se busca legitimar los sistemas médicos tradicionales o bien una burocratización del conocimiento médico local, mientras, a nivel local, la legitimación de las prácticas tradicionales responde más a las creencias, actitudes y expectativas de las personas enfermas. Por tanto, los estudios de corte positivista, como los analizados en este trabajo, en los que se dibuja una imagen de lo que es la asistencia basada en la profesionalización de las practicas médicas tradicionales, corren el riesgo de oscurecer gran cantidad de prácticas que influyen muy directamente en la salud de las personas de la comunidad pero que están ocultas al no haber pasado por los procesos de profesionalización y registro.

En los proyectos analizados, una vez identificados los sanadores, se evalúan sus prácticas. Esta evaluación se hace bajo las categorías propias de la biomedicina (tipo de enfermedad, signos, síntomas, diagnóstico, tratamiento, etc.). Los textos de Banerjee et al. (2000), Brouwer et al. (1998), Colvin et al. (2003), Edginton, Sekatane, Goldstein (2002), Haper et al. (2004) y Wilkinson, Gcabashe, Lurie (1999) se centran en el análisis del número de pacientes que son atendidos por los sanadores, el tipo de enfermedades que tratan, sus habilidades y conocimientos para detectar los signos y síntomas de la TBC. Esta fase es central para la problematización de la situación. Mediante el análisis de las capacidades de los sanadores se detecta su idoneidad como agentes capaces de llevar a cabo la supervisión de los tratamientos antituberculosos. Lo proyectos de Pronyk et al. (2001), Salaniponi et al. (2000), Sanou et al. (2004) y Steen, Mazonde $(1998,1999)$ analizaron las causas del retraso en el diagnóstico y tratamiento. Por ejemplo, en los artículos de Steen aparece un análisis descriptivo del comportamiento de las personas con TBC en su búsqueda de la sanación así como las creencias y actitudes que pueden provocar un retraso en la instauración de un tratamiento médico. Estos estudios complementan a los anteriores y concluyen culpabilizando a los sanadores del retraso en el diagnóstico precoz e instauración del tratamiento de la TBC: "En este estudio se ha encontrado una fuerte asociación entre pacientes atendidos por sanadores tradicionales o sanadores espirituales y el aumento del retraso total y el retraso en el contacto con servicios de salud" (Steen, Mazonde, 1998, p.632).

En estos proyectos con la sanción de ciertas prácticas y la potenciación de otras se genera un nuevo conocimiento médico supuestamente adaptado a las condiciones locales 
pero que refuerza o fuerza la introducción del modelo biomédico. Por ejemplo Banerjee et al. (2000), con una investigación realizada por personal del distrito médico de la zona, mediante entrevistas y cuestionarios, estudiaron el número de pacientes que eran atendidos por sanadores tradicionales, el tipo de enfermedades que trataban, la explicación local a las causas de las enfermedades y cómo la tos y la TBC eran percibidos e interpretados por el sistema médico tradicional. En la discusión del artículo los autores resaltan que el hallazgo más importante de la investigación había sido el conocimiento sobre las actividades que población local y sanadores desarrollaban ante la TBC. Los autores plantean como propuesta futura utilizar esta información para adiestrar a los sanadores en medidas biomédicas de control de la enfermedad, en concreto, supervisar el tratamiento antituberculoso. Harper et al. (2004) realizaron una valoración de los conocimientos biomédicos que los sanadores habían adquirido tras ser sometidos a un programa de entrenamiento. De los sanadores se esperaba que adquirieran un papel crucial en la prevención de la TBC, en la identificación de signos y síntomas y en la supervisión del tratamiento. Las investigaciones publicadas por Colvin et al. (2003), Harper et al. (2004), Peltzer, Mngqundaniso, Petros (2006a) o Wilkinson, Gcabashe, Lurie (1999), nos muestran, ya en su título ("Contribution of traditional healers to a rural tuberculosis control programme en Hlabisa, South Africa", "Traditional healers participate in tuberculosis control in The Gambia" o "Traditional healers as tuberculosis treatment supervisors: precedent and potencial"), la intencionalidad del estudio de integrar a los sanadores en los programas nacionales biomédicos de control de la TBC. Podemos concluir que los sistemas de entrenamiento, aunque afirman ajustarse a las circunstancias locales, enfatizan el interés por inocular el modelo biomédico frente a las ideas, practicas y condiciones locales.

La fase final que he identificado en los proyectos de colaboración con los sanadores autóctonos y que en cierta forma aglutina y es consecuencia de las anteriores, es lo que la OMS ha definido como integración de la medicina tradicional (WHO, 1978, 2002). Tras identificar los actores locales, valorar sus prácticas y proponer el modelo de actuación a seguir, los definidos como sanadores tradicionales se encuentran en condiciones óptimas para ser integrados, en una posición subsidiaria, en el sistema de atención sanitaria de la TBC. En todos los proyectos analizados, el concepto de integración se refiere, en realidad, a la medicalización de las prácticas de los sanadores, entendida como el desarrollo por parte de los agentes autóctonos de habilidades y conocimientos biomédicos tales como el reconocimiento de síntomas, remisión de personas afectadas a hospitales o supervisión del tratamiento antituberculoso. En el artículo de Colvin et al. (2003), por ejemplo, se afirma que el estudio ha sido puesto en marcha para "evaluar la aceptabilidad y eficacia de los sanadores tradicionales como supervisores del tratamiento para la tuberculosis" (p.87). Igualmente en el artículo de Harper et al. (2004, p.1267) se concluye: "Los sanadores tradicionales ocupan un lugar privilegiado en la salud de los países en desarrollo. Con una motivación y educación adecuadas, ellos pueden desempeñar un rol muy positivo en el tratamiento y remisión temprana de las personas sospechosas de padecer tuberculosis".

Estos son algunos de los ejemplos que nos muestran la intencionalidad de las investigaciones en sí y por extensión de los programas de acción sanitaria a los que pertenecen. En ninguno de los trabajos analizados se tiene en cuenta el contexto sociocultural en el 
que cobra sentido las prácticas y conocimientos locales. De hecho, no aparece estudio social previo ni referencias bibliográficas a otros autores que, desde otras disciplinas, han estudiado los factores socioculturales que han influido en el control de la TBC (Vecchiato, 1997). Tal y como se deduce de estas publicaciones, los proyectos a pesar de sus equívocos títulos son, en realidad, una manera de 'captar' agentes para la puesta en marcha de la terapia farmacológica antituberculosa. Tratamiento que necesita un alto grado de adhesión al requerir de las personas afectadas la ingesta sistemática de una combinación de antibióticos durante un periodo medio de entre seis y ocho meses. Además de hacer de los sanadores autóctonos un personal entrenado en labores propias de la biomedicina, tales como identificación y distinción de síntomas sugerentes de TBC, detección de casos nuevos y remisión a centros sanitarios.

Tabla 1 - Asistentes a la reunión de la OMS sobre promoción y desarrollo de la medicina tradicional. Ginebra, 28 de noviembre -2 de diciembre de 1977

\begin{tabular}{ll}
\hline $\begin{array}{l}\text { Expertos de los principales sistemas } \\
\text { de medicina tradicional }\end{array}$ & $\begin{array}{l}\text { Miembros del Grupo de Trabajo de la } \\
\text { Sede de la OMS sobre Medicina Tradicional }\end{array}$ \\
\hline $\begin{array}{l}\text { Dr. M. Badri, Departamento de Psicología de la } \\
\text { Universidad de Kartum, Sudán }\end{array}$ & $\begin{array}{l}\text { Dr. V. Fattorusso, División de Sustancias } \\
\text { Profilácticas, Terapéuticas y de Diagnóstico }\end{array}$ \\
\hline $\begin{array}{l}\text { Dr. D.M.R.D. Kissanayake, Comisionado de } \\
\text { Ayurveda, Colombo, Sri Lanka }\end{array}$ & $\begin{array}{l}\text { Dr. T. Harding, Médico Principal, División de } \\
\text { Salud Mental }\end{array}$ \\
\hline $\begin{array}{l}\text { Dr. F.F. Kao, Director del Instituto Superior de } \\
\text { Investigación sobre Ciencias y Medicina Asiáticas, } \\
\text { New York, EEUU }\end{array}$ & $\begin{array}{l}\text { Dr. I. Khan, Médico Principal, División de Salud } \\
\text { Mental }\end{array}$ \\
\hline $\begin{array}{l}\text { Dr. D.N. Lantum, Coordinador del Servicio de } \\
\text { Salud Pública, Yaundé, Camerún }\end{array}$ & Dr. T. a. Lambo, Director General Adjunto \\
\hline $\begin{array}{l}\text { Dr. X. Lozoya, Centro para el Estudio de la Plantas } \\
\text { Medicinales (IMEPLAM), México D.F., México }\end{array}$ & $\begin{array}{l}\text { Dr. A. Mangay Maglacas, Especialista de } \\
\text { Enfermería, División de Formación y } \\
\text { Perfeccionamiento del Personal de Salud }\end{array}$ \\
\hline $\begin{array}{l}\text { Hakim M. A. Razack, Asesor Adjunto (Unani), } \\
\text { Gobierno de la India, Nueva Delhi, India }\end{array}$ & $\begin{array}{l}\text { Dr. H. Nakajima, Jefe, Política y Gestión } \\
\text { Farmacéutica }\end{array}$ \\
\hline $\begin{array}{l}\text { Prof. M.D. Sayed, Pofesor de Farmacognosia, } \\
\text { Universidad de El Cairo, Egipto. }\end{array}$ & $\begin{array}{l}\text { Dr. N. Sartorius, Director, División de Salud } \\
\text { Mental }\end{array}$ \\
\hline & $\begin{array}{l}\text { Dr. I. Tabibzadeh, Médico, División de } \\
\text { Fortalecimiento de los Servicios de Salud }\end{array}$ \\
\hline & $\begin{array}{l}\text { Dr. M. Torfs, Jefe de Área de Programa, } \\
\text { Tecnología Apropiada para la Salud }\end{array}$ \\
\hline
\end{tabular}

Fuente: World Health Organization, The promotion and development of traditional medicine: report of a WHO meeting, 1978.

\section{Conclusión}

Como he tratado de exponer en este artículo, hablar de colaboración o de integración de las denominadas medicinas tradicionales y sus representantes en las campañas biomédicas de desarrollo puede generar falsas expectativas si se piensa como una comprensión, por parte de las agencias y agentes biomédicos, respeto y potenciación de los saberes médicos 
locales o autóctonos per se. Esta visión subalterna de los sistemas autóctonos de sanación se nos muestra ya desde la propia conceptualización de la tarea a acometer, es decir, en las estrategias de actuación definidas y consensuadas por las agencias internacionales, y se nos evidencia de forma clarificadora cuando analizamos la realidad práctica o sobre el terreno de estas campañas. O dicho de otra manera, cuando se pasa de lo formulado a lo realizado.

El análisis de las campañas y estrategias internacionales que se sustenta en una colaboración y promoción de lo tradicional plantea una serie de cuestiones cruciales en el análisis crítico de la biomedicina contemporánea y de la industria del desarrollo: ¿Qué prácticas/conocimientos locales son interpretados como tradicionales y utilizados por las campañas biomédicas de desarrollo? ¿En qué lugar queda lo local u autóctono en la relación biomedicina/medicinas tradicionales?

La revisión crítica de los artículos biomédicos referidos a campañas de colaboración con sanadores tradicionales en el control de la TBC en el África subsahariana entre 1996 y 2006, nos ha permitido comprender algunas formulaciones sobre el terreno de la promoción de lo tradicional. Estas campañas están basadas en las estrategias propuestas por la OMS, sustentadas en el análisis científico-médico de lo tradicional y obstinadas en generar una idea de las medicinas tradicionales ajustada a los preceptos del paradigma biomédico. Como nos muestran los artículos analizados, las agencias internacionales generan categorías discursivas como medicina tradicional, sanador tradicional, proceso de integración que muestran una visión parcial y jerárquica de los conocimientos locales, generando desigualdad de los agentes locales con respecto a los centros de decisión (Latour, 1992, p.205-243) de las agencias internacionales de salud. El concepto sanador tradicional es utilizado como un eufemismo 'políticamente' correcto para introducir, sin una revisión crítica de su eurocentrismo, la biomedicina. Cuando las agencias internacionales analizadas, que o bien gestionan directamente las campañas o bien sirven como apoyo gerencial e ideológico a programas nacionales, planifican actuaciones orientadas a potenciar lo tradicional, lo hacen, según nos muestran los proyectos analizados, mediante el análisis científico de ciertas facetas de los saberes médicos locales y de las prácticas de algunos de los definidos como responsables locales en salud. También, mediante la instauración de programas de entrenamiento de los sanadores tradicionales orientados a que estos cambien sus conductas cuando abordan temas relacionados con el objetivo de la campaña, en nuestro caso la TBC.

Aunque los estudios recientes parecen partir de propuestas más novedosas en el campo del desarrollo, como es el análisis de las medicinas tradicionales y sus representantes, no se considera el contexto sociocultural de los saberes y prácticas autóctonos y se reproduce una relación de hegemonía de la biomedicina frente a lo local que queda en una posición subalterna. Esta situación nos muestra la incapacidad de trascender la planificación biocéntrica en la que se sustenta la gestión internacional de la salud. Comités de expertos sumidos en el paradigma biomédico, la grávida herencia colonial (como mostraba en el primer apartado), la falta de experiencia en la gestión de la salud local o la infravaloración de los conocimientos locales en salud son factores que pueden explicar tal situación.

Con este trabajo no pretendo restar importancia a las acciones de las agencias internacionales ni banalizar o sobrevalorar las prácticas tradicionales. El propio concepto de integración no tiene porqué suponer la desaparición de las medicinas tradicionales. De 
hecho, paradójicamente, en un entorno médico cada vez más globalizador, este tipo de medidas pueden suponer la pervivencia de ciertas prácticas, como puede ser el caso de la acupuntura que se está instalando en sistemas médicos occidentales. No se trata de sostener una visión romántica de las prácticas tradicionales. La cuestión de la interrelación entre lo local y lo global es compleja y deja muchos debates abiertos. ¿Politización de la salud o potenciación de lo autóctono? ¿Extensión del imperio biomédico o autonomía de lo tradicional? Según los proyectos y estrategias analizados en relación a la TBC en el África subsahariana, las políticas actuales subsumen la tradición a la racionalidad universal del modelo de desarrollo biomédico. En los proyectos se nos muestra al sanador como recurso y no como obstáculo, lo que puede generar a nivel discursivo la idea de respeto, cuando en el nivel práctico observamos que los sanadores se convierten en figuras de control, que quedan relegados a una posición subalterna en el entramado biomédico de las campañas sanitarias. Por tanto, la tradición es entendida como algo aislado, algo tangible desligado del sistema sociocultural en el que cobra sentido. Estas prácticas e ideas y la dificultad para cambiarlas también responden a la burocratización a la que se ven sometidos los proyectos económico-políticos de desarrollo neoliberales.

Algunos planteamientos contemporáneos proponen la necesidad de transformaciones epistemológicas para la recuperación del punto de vista de los actores locales (Medina, 2005) o de análisis socioculturales de la planificación del desarrollo (Santander, 1997; Cernea, 1985). Otros planteamientos analíticos buscan transcender la causalidad biológica de la enfermedad y se centran en las representaciones culturales de la misma (Haro, 2000). Incluso, desde la propia industria del desarrollo, se ha propuesto la síntesis de los factores epidemiológicos y culturales para solucionar el conflicto entre la medicina occidental y la tradicional (Sanz, 1995a, 1995b) y abrir nuevas y verdaderas fórmulas de diálogo.

Con este trabajo no he pretendido generar soluciones cerradas a la problemática tratada, más bien abrir un campo de análisis para la reflexión partiendo de un mejor conocimiento, tanto histórico como socio-cultural, del uso de la medicina tradicional y concepciones subyacentes al término y a las practicas que hoy en día inspira.

\section{NOTAS}

* Trabajo realizado para la obtención del Diploma de Estudios Avanzados (DEA) en el programa de doctorado Salud: Antropología e Historia, bajo la dirección de la profesora Rosa María Medina Doménech.

${ }^{1}$ Un buen ejemplo lo proporcionan los azande, donde los oráculos tenían una influencia directa en la organización política local mediante la división de la población en clases, lo que les confería poder y autoridad (McLeod, 1972, citado en Feierman, 1985, p.75).

${ }^{2}$ En esta y demás citas de los textos en otros idiomas, la traducción es libre.

${ }^{3}$ B. Velimirovic (1990) señala que el concepto de medicina tradicional, utilizado en la estrategia de 1978, dificultó la aplicación de estas medidas. Con respecto al problema que plantea la homogenización del término sanador tradicional en las campañas sanitarias de desarrollo, ver Van der Geest, 1997 y McMillen, 2004.

${ }^{4}$ Para conocer las diferencias en la gestión del padecimiento del sistema médico chino con respecto a otros sistemas médicos como el biomédico, véase Kleinman, 2000. Sobre el pluralismo médico africano y la variabilidad y características de las prácticas médicas tradicionales, ver Feierman, 1985; Freeman, Montsei, 1992; Prins, 1991.

${ }^{5}$ Entre los proyectos de evaluación analizados en este trabajo podemos destacar King, 2000,2002 y Richter, 2003. 


\section{REFERENCIAS}

ANDERSON, Warwick.

Postcolonial technoscience. Social Studies of Science, London, v.32, n.5-6, p.643-658. 2002.

ARNOLD, David.

Medicine and colonialism. In: Bynum, William; Porter, Roy (Ed.). Companion encyclopaedia of the history of medicine. London: Routledge. p.1393-1416. 1993.

BANERJEE, A. et al.

Local perceptions of tuberculosis in a rural district in Malawi. International Journal of Tuberculosis and Lung Disease, Paris, v.4, n.11, p.1047-1051. 2000 .

BRAY, Francesca.

Chinese medicine. In: Bynum, William; Porter, Roy (Ed.). Companion encyclopaedia of the history of medicine. London: Routledge. p.728-54. 1993.

BROUWER, J.A. et al.

Traditional healers and pulmonary tuberculosis in Malawi. International Journal of Tuberculosis and Lung Disease, Paris, v.2, n.3, p.231-234. 1998.

CERNEA, Michael.

El conocimiento de las ciencias sociales y las políticas y los proyectos de desarrollo. In: Cernea, Michael (Ed.). Poner primero a la gente. México: Fondo de Cultura Económica. p.25-66. 1985.

COLVIN, M. et al.

Contribution of traditional healers to a rural tuberculosis control programme in Hlabisa, South Africa. International Journal of

Tuberculosis and Lung Disease, Paris, v.7, n.9, p.86-91. 2003.

COMELLES, Josep.

El regreso de las culturas: diversidad cultural y práctica médica en el siglo XXI. In: Fernández, Gerardo (Ed.). Salud e interculturalidad en América Latina: perspectivas antropológicas. Quito: Abya-Yala. p.17-30. 2004.

EDGINTON, M.E.; SEKATANE, C.S; GOLDSTEIN, S.J.

Patients' beliefs: do they affect tuberculosis control? - a study in a rural district of South Africa. International Journal of Tuberculosis and Lung Disease, Paris, v.6, n.12, p.1075-1082. 2002.

ESCOBAR, Arturo.

Planning. In: Sachs, Wolfgang (Ed.). The development dictionary: a guide to knowledge as power. London: Zed Books. p.216-233. 1992.

FEIERMAN, Steven.

Struggles for control: the social roots of health and healing in modern Africa. African Studies Review, Amherst, v.28, n.2-3, p.73-147. 1985.
FREEMAN, Melvyn; MOTSEI, Mmatshilo. Planning health care in South Africa: is there a role for traditional healers? Social Science and Medicine, Elmsford, v.34, n.11, p.1183-1190. 1992.

GODFREY-FAUSSETT, P.; AYLES, H. Can we control tuberculosis in high HIV prevalence settings? Tuberculosis (Edinburgh), Edinburgh, v.83, n.1-3, p.68-76. 2003.

GREEN, Edward.

Can collaborative programs between biomedical and african indigenous health practitioners succeed? Social Science and Medicine, Elmsford, v.27, n.11, p.1125-1130. 1988.

GREEN, Edward; MAKHUBU, Lydia.

Traditional healers in Swaziland: toward improved cooperation between the traditional and modern health sectors. Social Science and Medicine, Elmsford, v.18, n.12, p.1071-1079. 1984.

HARO, Jesús A.

Cuidados profanos: una dimensión ambigua en la atención de la salud. In: Perdiguero, Enrique; Comelles, Josep M. (Ed.). Medicina y cultura: estudios entre la antropología y la medicina. Barcelona: Bellaterra. p.101-161. 2000.

HARPER, M.E. et al.

Traditional healers participate in tuberculosis control in The Gambia. International Journal of Tuberculosis and Lung Disease, Paris, v.8, n.10, p.1266-1268. 2004.

ILIFFE, John.

África: historia de un continente. Cambridge: Cambridge University Press. 1998.

KING, Rachel.

Collaboration with traditional healers in HIV/ AIDS prevention and care in sub-Saharan Africa: a literature review. Geneva: Unaids. (UNAIDS/ 00.29E). 2000.

KING, Rachel.

Ancient remedies, new disease: involving traditional healers in increasing access to AIDS care and prevention in East Africa. Geneva: Unaids. (UNAIDS Best Practice Collection). Disponible en: http://www.unaids.org/ publications/documents/care/general/JC761AncientRemedies-E.pdf). Acceso en: 15 ene. 2007. 2002.

KLEINMAN, Arthur.

Suffering in China and the West: the challenge of an interpersonal locus of experience to the hipertrophy of individual autonomy in health. 
In: Woodeward, John; Jütte, Robert (Ed.). Coping with sickness: perspectives on health care, past and present. Sheffield: European Association for the History of Medicine and Health Publications. p.43-52. 2000.

KNIPPER, Michael.

El reto de la 'medicina intercultural' y la historia de la 'medicina tradicional' indígena contemporánea. In: Fernández, Gerardo (Ed.). Salud e interculturalidad en América Latina: antropología de la salud y crítica intercultural. Quito: Abya-Yala. p.413-432. 2006.

KOCHI, Arata.

The global tuberculosis situation and the new control strategy of the World Health

Organization. Tubercle, New York, v.72, n.1-6. 1991.

LAST, Murray.

The professionalization of indigenous healers. In: Johnson, Thomas; Sargent, Carolyn (Ed.). Medical anthropology: contemporary theory and method. New York: Greenwood. p.349-366. 1990.

LATOUR, Bruno.

Ciencia en acción. Barcelona: Labor. 1992.

MARKS, Shula; ANDERSON, Neil.

Typhus and social control: South Africa, 1917-1950. In: McLeod; Roy; Lewis, Milton (Ed.). Disease, medicine and empire: perspectives on Western medicine and the experience of European expansion. London: Routledge. p.257-283. 1988.

MCLEOD, Malcom.

Oracles and accusations among the azande. In: Singer, André; Street, Brian (Ed.). Zande themes. Oxford: Blackwell. p.158-72. 1972.

MCMILLEN, Heather.

The adapting healer: pioneering through shifting epidemiological and sociocultural landscapes. Social Science and Medicine, Elmsford, v.59, n.5, p.889-902. 2004.

MEDINA, Rosa M.

La historia de la medicina en el siglo XXI: una visión poscolonial. Granada: Universidad de Granada. 2005.

MEDINA, Rosa M.

Paludismo, explotación y racismo científico en Guinea Ecuatorial (1900-1939). In: Rodríguez, Esteban et al. La acción médico-social contra el paludismo en la España metropolitana y colonial del siglo XX. Madrid: CSIC. p.383-427. 2003.

MENÉNDEZ, Eduardo L.

El saber popular como proceso de trasformación: tipos de articulación entre la biomedicina y la medicina popular. In:
González Alcantud, Jose A.; Rodríguez Becerra, Salvador (Ed.). Creer y curar: la medicina popular. Granada: Diputación de Granada. p.31-61. 1996.

OFFIONG, Daniel.

Traditional healers in the nigerian health care delivery system and the debate over integrating traditional and scientific medicine.

Anthropological Quarterly, Washington, v.72, n.3, p.118-130. 1999.

PACKARD, Randal.

Post-colonial medicine. In: Cooter, Roger; Pickstone, John (Ed.). Medicine in the twentieth century. Amsterdam: Harwood Academic Publishers. p.97-112. 2000.

PACKARD, Randal.

Industrialization, rural poverty, and tuberculosis in South Africa, 1850-1950. In: Feierman, Steven; Janzen, John (Ed.). The social basis of health and healing in Africa. Berkeley: University of California Press. p.104-130. 1992.

PELTZER, K.; MNGQUNDANISO, N.; PETROS, G.

A controlled study of an HIV/AIDS/STI/TB: intervention with traditional healers in KwaZulu-Natal, South Africa. AIDS and Behavior, New York, v.10, n.6, p.683-690. 2006a.

PELTZER, K.; MNGQUNDANISO, N.; PETROS, G.

HIV/AIDS/STI/TB knowledge, beliefs and practices of traditional healers in KwaZuluNatal, South Africa. AIDS Care, Oxford, v.18, n.6, p.608-613. 2006b.

PIGG, Stacy.

Acronyms and effacement: traditional medical practitioners (TMP) in international health development. Social Science and Medicine, Elmsford, v.41, n.1, p.47-68. 1995.

PRINS, Gwyn.

¿Pero de qué enfermedad se trataba?: el estado actual de los estudios sobre la salud y curación en África. Dynamis, Granada, v.11, p.361-385. 1991.

PRONYK, R.M. et al.

Assessing health seeking behaviour among tuberculosis patients in rural South Africa. International Journal of Tuberculosis and Lung Disease, Paris, v.5, n.7, p.619-627. 2001.

RICHTER, Marlise.

Traditional medicines and traditional healers in South Africa: discussion paper prepared for the Tratment Action Campaign an AIDS Law Project. Disponible en: http://www.tac.org.za/ Documents/ResearchPapers/

Traditional_Medicine_briefing.pdf. Acceso en: 27 ene. 2007. 2003. 
RUBINSTEIN, Robert; LANE, Sandra. International health and development. In: Johnson, Thomas; Sargent, Carolyn (Ed.). Medical anthropology: a handbook of theory and method. New York: Greenwood. p.367-390. 1990.

RUIZ-GIMÉNEZ, Itziar.

Las 'buenas intenciones': intervención humanitaria en África. Barcelona: Icaria. 2003.

SALANIPONI, F.M. et al.

Care seeking behaviour and diagnostic processes in patients with smear-positive pulmonary tuberculosis in Malawi. International Journal of Tuberculosis and Lung Disease, Paris, v.4, n.4, p.327-332. 2000.

SANOU, A. et al.

Access and adhering to tuberculosis treatment: barriers faced by patients and communities in Burkina Faso. International Journal of Tuberculosis and Lung Disease, Paris, v.8, n.12, p.1479-1483. 2004.

SANTANDER, M. Teresa.

La transferencia tecnológica: su complejidad en la periferia. In: Rodríguez, Francisco; Medina, Rosa; Sánchez, Jesús (Ed.). Ciencia, tecnología y sociedad: contribuciones para una cultura de paz. Granada: Universidad de Granada/ Instituto de Estudios Por la Paz y los Conflictos. p.273-288. 1997.

SANZ, Juan.

Biomedicina frente a medicina tradicional masai: análisis de un conflicto. Jano, Madrid, v.68, n.1120, p.57-61. 1995a.

SANZ, Juan.

El proyecto de desarrollo y el contexto cultural. Jano, Madrid, v.68, n.1120, p.63-68. 1995b.

SILVA, K.T.

Malaria eradication as a legacy of colonial discourse: the case of Sri Lanka. Parassitologia, Roma, v.36, n.1-2, p.149-163. 1994.

STEEN, T.W.; MAZONDE, G.N. Ngaka ya setswana, ngaka ya sekgoa or both?: health seeking behaviour in Batswana with pulmonary tuberculosis. Social Science and Medicine, Elmsford, v.48, n.2 , p.163-172. 1999.

STEEN, T.W.; MAZONDE, G.N.

Pulmonary tuberculosis in Kweneng district, Botswana: delays in diagnosis in 212 smearpositive patients. International Journal of
Tuberculosis and Lung Disease, Paris, v.2, n.8, p.627-634. 1998.

VAN DER GEEST, Sjaak.

Is there a role for traditional medicine in basic health services in Africa?: a plea for a

community perspective. Tropical Medicine and International Health, Oxford, v.2, n.9, p.903-911. 1997.

VAN DER GEEST, Sjaak; SPECKMANN, John D.; STREEFLAND, Pieter $\mathrm{H}$.

Primary health care in a multi-level perspective: towards a research agenda. Social Science and Medicine, Elmsford, v.30, n.9, p.1025-1034. 1990.

VECCHIATO, Norbert.

Sociocultural aspects of tuberculosis control in Ethiopia. Medical Anthropology Quarterly, Washington, v.11, n.2, p.83-201. 1997.

VELIMIROVIC, Boris.

Is integration of traditional and western medicine really possible? In: Coriel, Jeannine; Mull, Dennis (Ed.). Anthropology and primary health care. Boulder: Westview Press. p.51-78. 1990.

WHO.

World Health Organization. WHO traditional medicine strategy, 2002-2005. Geneva: WHO. 2002.

WHO.

World Health Organization. Traditional healers as community health workers: a review of projects using traditional healers as community health workers. Geneva: WHO. 1991.

WHO.

World Health Organization. The promotion and development of traditional medicine: report of a WHO meeting. Geneva: WHO (WHO Tech. Rep. Ser.). 1978.

WHO; UNICEF.

World Health Organization; Unicef. Primary health care: report of the International Conference on Primary Health Care. Alma Ata. Geneva: WHO. 1978.

WILKINSON, D.; GCABASHE, L.; LURIE, M. Traditional healers as tuberculosis treatment supervisors: Precedent and potential. International Journal of Tuberculosis and Lung Disease, Paris, v.3, n.9, p.838-842. 1999.

\section{$\rightarrow \rightarrow \rightarrow<<<$}

